Copyright (C) 2007 Institute of Electrical and Electronics Engineers, Inc. All rights reserved. Personal use of this material, including one hard copy reproduction, is permitted. Permission to reprint, republish and/or distribute this material in whole or in part for any other purposes must be obtained from the IEEE. For information on obtaining permission, send an e-mail message to stds-ipr@ieee.org. By choosing to view this document, you agree to all provisions of the copyright laws protecting it. Individual documents posted on this site may carry slightly different copyright restrictions. For specific document information, check the copyright notice at the beginning of each document. 


\title{
Spam Classification Using Adaptive Boosting Algorithm
}

\author{
ABM Shawkat $\mathrm{Ali}^{1}$ and Yang Xiang ${ }^{2}$ \\ ${ }^{I}$ School of Computing Sciences \\ ${ }^{2}$ School of Management and Information Systems \\ Central Queensland University \\ Rockhampton, QLD 4702, Australia \\ E-mail: \{s.ali,y.xiang\}@cqu.edu.au
}

\begin{abstract}
Spam is no doubt a new and growing threat to the Internet and its end users. This paper investigates current approaches for blocking spam and proposes a new spam classification method by using adaptive boosting algorithm. Experiment is carried out to evaluate the results of spam filtering. We find adaptive boosting algorithm is an effective approach to solve the spam problem. We also find that default method in WEKA such as DecisionStump is not actually the best associated algorithm to filter spam. After comparing DecisionStump, J48, and NaiveBayes we conclude $J 48$ is the most suitable associated algorithm to filter spam with high true positive rate, low false positive rate and low computation time.
\end{abstract}

Keywords: Spam, filtering, Boosting algorithm.

\section{Introduction}

Spam has grown from being a minor nuisance to a worldwide electronic epidemic in the past years. The percentage of the total Internet email messages identified as spam has increased from 45\% March 2003 to $62 \%$ in February 2004 as reported by a leading antispam vendor, Brightmail [5]. The exponential growth of spam over recent years has also brought exponential financial costs and security risks to organizations world wide. According to a study conducted by Ferris Research, spam cost US businesses $\$ 10$ billion in 2003, a $12 \%$ increase from the $\$ 8.9$ billion from the previous year [10]. The cost to businesses include the need to purchase anti-spam solutions; additional network routers and servers to support the giant waves of incoming messages; cover the costs of lost employee productivity, which is estimated to be an average of
US\$874 per employee each, year to US organizations according to Nucleus Research [16]; amounting to the cost is the legal liability placed on employers failing to protect their employees from offensive content contained in spam.

There are many definitions of spam in the literature. Due to the majority of spam containing content of commercial nature, many legislative bodies and antispam vendors have defined spam as any "unsolicited commercial email/messages/communications". respectively $[3,8]$. The messages vary from short messaging service (SMS), instant messaging (IM), electronic voice calls and emails. In addition, "any unwanted electronic messages" may contain content of non-commercial nature, such as promoting a religion or simply chain-letters choking network resources. Therefore, in this paper, we define spam as 'any unwanted email', whether sent in bulk or singly to anyone and/or anywhere.

Efforts by anti-spam vendors and academia have produced an array of technologies to counter the spam problem. These technologies range from classification and filtering technology installed on corporate networks or ISPs to collaborative anti-spam network involving end-users from around the world. In this paper, we propose a new method by using adaptive boosting algorithm to detect and filter spam. Experiment is carried out to evaluate the results of spam filtering. We find adaptive boosting algorithm is an effective approach to solve the spam problem. We also find that classic method in WEKA [14] such as $\mathrm{J} 48$ is not actually the best associated algorithm to filter spam. After comparing DecisionStump [14] J48 [14], and NaiveBayes [12] we conclude DecisionStump is the most suitable associated algorithm to filter spam with high true positive rate, low false positive rate and low computation time. 
The rest of the paper is organized as follows. Section 2 introduces current spam classification techniques. Section 3 presents the adaptive boosting algorithm. Section 4 presents the experiment details and the results. Section 5 discusses issues in spam filtering and future work. Section 6 concludes this paper.

\section{Current Spam Classification Techniques}

Current spam classification techniques include neural network, Bayesian statistics, lexical analysis heuristic-based analysis, and signature-based analysis.

Neural network algorithm is a classical algorithm which has been successfully applied in the character and speech recognition, intrusion and virus detection and so on [17]. Neural network has an input layer which is the parameters input to the network, an output layer which gives the analysis result and one or more than more middle layers. The parameters include keywords in subject field, keywords in the body, domain names etc. The solutions using Neural Network include SurfControl E-mail Filter [20] and AntiSpam for SMTP [21].

Bayesian statistics bases on the idea that we are interested in a person's degree of belief in a particular event; that is the Bayesian probability of the event [11]. Key words in an email are analyzed by Bayesian statistic approach to give a probability of this mail being a spam. The Bayesian approach lets us combine our general background information we got from analyzing previous emails with the data we have collected from the current email in such a way that both aspects are given their proper importance. In this way, we determine an appropriate degree of belief about whether, when we see the word again, it will be in a spam. The software products using Bayesian approach include Brightmail Anti-Spam [5], MailSweeper ${ }^{\text {TM }}$ Business Suite [6], and Cloudmark Authority [7].

Lexical analysis searches for key words or phrases in the entire email including message envelop, header, subject, body, attachments and HTML tags to judge if the email is a spam or not [4]. Keys words and phrases occurring frequently in the spam are mainly associated with offer to purchase something, offer to use services, solicitation to visit a web site etc [1]. Lexical analysis is used in these products: Tumbleweed Email Firewall [23], Brightmail Anti-Spam [5], SurfControl E-mail Filter [20].

Heuristic approach will generate patterns for spam after analysing large amount of spam [9]. Patterns are the common characteristics of spam, such as the usage of mixed foreign character set, image likes which are server queries, mix of different obscure and/or nonprintable characters, different coding methods etc [1]. Solutions using heuristics-based analysis include Brightmail Anti-Spam [5], Tumbleweed Email Firewall [23], IM Message Inspector [27], SPS [22], McAfee SpamKiller [15] etc.

The signature of a spam is the hash value of this spam calculated by some hash algorithm which inspect the part of the spam message and generate a hash signature. Spam is identified by comparing their signatures with the signatures in the database. A good hash-signature system should tolerate the slight variation of the spam [4]. Typical products include Cloudmark SpamNet [7], IM Message Inspector [27].

\section{Adaptive Boosting Algorithm}

Boosting is a well established method in the machine learning community for improving the performance of any learning algorithm. The boosting algorithms were first presented by Schapire [18] and Freund [24]. Continuing this research they introduced a new generation of boosting algorithm called Adaptive Boosting (AdaBoost) [2, 19]. Schapire and Freund argued that this new boosting algorithm has certain properties which make it more practical and easier to implement than its predecessors [25].

AdaBoost.M1 and AdaBoost.M2 were developed by Schapire and Freund from their AdaBoost algorithm. For binary classification problems the two versions are equivalent, differing only in the way they handle problems with more than two classes [26]. AdaBoost.M1 has access to a learning algorithm (which the developers generically title WeakLearn) which it calls repeatedly with distributions over the training set. AdaBoostM1 consider the distribution $D_{t}$ as follows:

$D_{t+1(i)}=\frac{D_{t}(i)}{Z_{t}} \times \begin{cases}\beta_{t} & \text { if } \mathrm{h}_{\mathrm{t}}\left(x_{i}\right)=y_{i} \\ 1 & \text { otherwise }\end{cases}$

Where $Z_{t}$ is a normalization constant (chosen so that $D_{l+1}$ will be distribution.

WeakLearn calculates a hypothesis, or classifier, that attempts to correctly classify all instances of the test data. As described previously, examples that are incorrectly classified are given greater weighting for the next pass. Finally, the boost algorithm combines all the hypotheses into one final hypothesis [26].

$h_{f i n}(x)=\underset{y \in Y}{\arg \max } \sum_{t: h_{t}(x)=y} \log \frac{1}{\beta_{t}}$ 
It is noted that sequences of $m$ instances are $\left\langle\left(x_{1}, y_{1}\right), \ldots,\left(x_{m}, y_{m}\right)\right\rangle$ with the target values $y_{i} \in Y=\{1, \ldots, k\}$.

\section{Experiment and Evaluation}

We tested AdaBoostM1 algorithm by using public spam corpus from [13]. Both classic algorithms without AdaBoostM1 and with AdaBoostM1 algorithm are tested.

\subsection{Classic Algorithms without AdaBoostM1}

If no AdaBoostM1 algorithm is used, we obtain the following results. Spam classification accuracy is shown in Table 4.1. From the table we can see that both Decision Stump and J48 are good algorithms which provide high accuracy rate when classifying spam. NaiveBayes is about $10 \%$ lower in accuracy rate compared to the previous two algorithms.

Table 4.1 Classification Accuracy Rate

\begin{tabular}{|l|l|l|}
\hline Decisionstump & $\mathrm{J} 48$ & Naivebayes \\
\hline 91.50 & 92.98 & 79.29 \\
\hline
\end{tabular}

Confusion matrix based analysis is shown in table 4.2. From the table we can see that NaiveBayes has the highest True Positive Rate (TPR). However, it performs poorly in False Positive Rate (FPR), which means it mistakenly classifies legitimate emails as spam. Both DecisionStump and J48 have good TPR which is around 0.9 and good FPR which is around 0.05 . They also have good True Negative Rate (TNR), which means the ability to classify nonspam.

Table 4.2 Confusion Matrix Based Analysis

\begin{tabular}{|l|l|l|l|l|}
\hline Algorithm & TPR & FPR & TNR & FNR \\
\hline Decisionstump & 0.87 & 0.05 & 0.95 & 0.13 \\
\hline J48 & 0.91 & 0.06 & 0.94 & 0.09 \\
\hline Naivebayes & 0.95 & 0.31 & 0.69 & 0.05 \\
\hline
\end{tabular}

Table 4.3 shows the computation time of each algorithm. We can find that the training time of J48 is the longest, which is 94.37 seconds, and is 12.3 times of DecisionStump and 5.8 times of NaiveBayes. Both DecisionStump and $\mathrm{J} 48$ perform well in testing time, which is below 0.03 seconds. The testing time of NaiveBayes is much slower than the previous two, which is nearly half second.
Table 4.3 Computation Time

\begin{tabular}{|l|lc|l|}
\hline \hline Algorithm & $\begin{array}{l}\text { Training } \\
(\text { Sec.) }\end{array}$ & $\begin{array}{l}\text { Testing Time } \\
(\text { Sec.) }\end{array}$ \\
\hline Decisionstump & 7.65 & 0.01 \\
\hline J48 & 94.37 & 0.03 \\
\hline Naivebayes & 16.04 & 0.58 \\
\hline
\end{tabular}

\subsection{Classic Algorithms with AdaBoostM1}

If AdaBoostM1 algorithm is used, we obtain the following results. Spam classification accuracy is shown in table 4.4. From the table we can see that both Decision Stump and J48 are good algorithms which provide high accuracy rate when classifying spam. If we compare table 4.1 and 4.4 we can find that the AdaBoostM1 increases the accuracy of $\mathrm{J} 48$ and decreases the accuracy of DecisionStump. This is a proof that J48 works better with AdaBoostM1 than DecisionStump. AdaBoostM1 does not affect the accuracy rate of NaiveBayes.

Table 4.4 Classification Accuracy Rate

\begin{tabular}{|l|l|l|}
\hline Decisionstump & $\mathrm{J} 48$ & Naivebayes \\
\hline 90.07 & 95.15 & 79.29 \\
\hline
\end{tabular}

Confusion matrix based analysis with AdaBoostM1 is shown in table 4.5. From the table we can see that NaiveBayes still has the highest True Positive Rate (TPR) and it still performs poorly in False Positive Rate (FPR). TPR, FPR and TNR of J48 are improved. However, all these values of DecisionStump are decreased. If we compare table 4.2 and table 4.5 , the conclusion is that the optimized algorithm is J48 with AdaBoostM1.

Table 4.5 Confusion Matrix Based Analysis

\begin{tabular}{|l|l|l|l|l|}
\hline Algorithm & TPR & FPR & TNR & FNR \\
\hline Decisionstump & 0.86 & 0.08 & 0.92 & 0.14 \\
\hline J48 & 0.93 & 0.04 & 0.96 & 0.07 \\
\hline Naivebayes & 0.95 & 0.31 & 0.69 & 0.05 \\
\hline
\end{tabular}

Table 4.6 shows the computation time of each algorithm with AdaBoostM1. We can find that the training time of J48 is significantly improved to only 6.84 seconds. It is much faster than the training time of each algorithm without AdaBoostMl. The testing time of J48 with AdaBoostM1 is also significantly reduced to only 0.01 second. This verifies our idea to use 
AdaBoostM1 to enhance the performance of spam classifier.

Table 4.6 Computation Time

\begin{tabular}{|l|l|l|}
\hline Algorithm & Training Time (Sec.) & $\begin{array}{l}\text { Testing } \\
\text { Time (Sec.) }\end{array}$ \\
\hline Decisionstump & 35.72 & 0.03 \\
\hline J48 & 6.84 & 0.01 \\
\hline Naivebayes & 0.61 & 0.22 \\
\hline
\end{tabular}

Table 5.1 Comparison between Different Spam Classification Techniques

\begin{tabular}{|c|c|c|}
\hline $\begin{array}{l}\text { Spam } \\
\text { Classification } \\
\text { Techniques }\end{array}$ & Advantages & Disadvantages \\
\hline $\begin{array}{l}\text { Neural } \\
\text { Network }\end{array}$ & $\begin{array}{l}\text { Effectively detect } \\
\text { the spam similar } \\
\text { with known spam } \\
\text { Can be customized } \\
\text { for enterprises by } \\
\text { using their own } \\
\text { data to train the } \\
\text { neural network }\end{array}$ & $\begin{array}{l}\text { It is difficult for } \\
\text { users to choose } \\
\text { input training } \\
\text { parameters. }\end{array}$ \\
\hline $\begin{array}{l}\text { Bayesian } \\
\text { Statistics }\end{array}$ & $\begin{array}{l}\text { Can be customized } \\
\text { for end users at the } \\
\text { desktop by training } \\
\text { the algorithm using } \\
\text { users' own data }\end{array}$ & $\begin{array}{l}\text { Not effective at } \\
\text { enterprise scale }\end{array}$ \\
\hline $\begin{array}{l}\text { Lexical } \\
\text { analysis }\end{array}$ & $\begin{array}{ll}\text { Easy to } & \text { be } \\
\text { customized } & \text { or } \\
\text { tuned } & \\
\end{array}$ & $\begin{array}{l}\text { Can not filter } \\
\text { spam which have } \\
\text { no text, just URLs } \\
\text { and images }\end{array}$ \\
\hline $\begin{array}{l}\text { Heuristic- } \\
\text { Based } \\
\text { Analysis }\end{array}$ & $\begin{array}{l}\text { Can deal with text- } \\
\text { base, graphics- } \\
\text { based and HTML } \\
\text { spam }\end{array}$ & $\begin{array}{l}\text { Heuristic rules are } \\
\text { complex. } \\
\text { It needs a dynamic } \\
\text { update service. }\end{array}$ \\
\hline $\begin{array}{l}\text { Signature- } \\
\text { Based } \\
\text { Analysis }\end{array}$ & Less false positive & $\begin{array}{l}\text { Sensitive to the } \\
\text { variation of spam. }\end{array}$ \\
\hline AdaBoostMI & $\begin{array}{l}\text { Greatly improving } \\
\text { classic algorithm, } \\
\text { such as } \mathrm{J} 48\end{array}$ & $\begin{array}{l}\text { AdaBoostMl is } \\
\text { unable to handle } \\
\text { weak hypotheses } \\
\text { with error greater } \\
\text { than } 1 / 2 \text {. }\end{array}$ \\
\hline
\end{tabular}

\section{Discussions}

Currently there is no any single spam classifier or combined spam classifiers that can not block spam with one hundred percent accuracy rate. Table 5.1 shows the comparison between different spam classification techniques. Compared to current spam classification techniques, AdaBoostM1 algorithm has the following advantages and disadvantages. While the advantages of proposed algorithm can be applied to direct spam filter applications, the disadvantages can be our future research work. Moreover, how we can select a unique classifier for AdaBoostM1 could be a upcoming research issue.

\section{Conclusion}

Spam is a non-trivial problem which brings more and more threats to people's work and life. The driving force for spammers to send unsolicited emails is the economic interest. This paper proposes a new spam classification algorithm. This algorithm can effectively classify spam messages according to our experiment. Our finding is that default method in WEKA such as DecisionStump is not actually the best associated algorithm to filter spam. After comparing DecisionStump, J48, and NaiveBayes we can draw a conclusion that $\mathrm{J} 48$ is the most suitable associated algorithm with AdaBoostM1 to filter spam with high true positive rate, low false positive rate and computationally less expensive.

\section{References}

[1] Aladdin Knowledge Systems, "Anti-Spam White Paper", http://www.esafe.com/pdf/esafe/esafe_antispam_whitepaper. pdf, 2004.

[2] A. Grove and D. Schuurmans, "Boosting in the limit: Maximizing the margin of learned ensembles," in Proceedings of the Fifteenth National Conference on Artifical Intelligence, pp. 692-699, 1998.

[3] Australian Spam Act 2003, http://www.noie.gov.au/publications/NOIE/spam/Act2003/, 2003.

[4] Blackspider Technologies Ltd., "MailControl Spam Technology Overview", http://www.blackspider.com/services-spam_datasheet.pdf, 2003.

[5] Brightmail Inc. http://www.brightmail.com, 2006.

[6] Clearswift Corporation, http://www.clearswift.com/, 2006.

[7] Cloudmark company, http://www.cloudmark.com/, 2006. 
[8] Commission of The European Communities, "Communication form the Commission to the European Parliament, The Council, The European Economic and Social Committee and the Committee of the Regions on unsolicited commercial communications or "spam", $\operatorname{COM}(2004) 28$ final, 2004.

[9] Eric Allman, "The FTC and Spam", ACM Queue, Vol. 1, No. 6, 2003.

[10] Ferris Research report: "Spam Control: Problems and Opportunities," http://www.ferris.com/rep/200301/report.pdf.

2003 ,

[11] Gary Robinson, "A Statistical Approach to the Spam Problem”, Linux Journal, Vol. 2003, No. 107, 2003.

[12] G.H. John and P. Langley, "Estimating continuous distributions in Bayesian classifiers," in Proceedings of the Eleventh Conference on Uncertainty in Artificial Intelligence, Morgan Kaufmann, San Mateo, CA, pp. 338$345,1995$.

[13] Hakank Spam

Data, http://www.hakank.org/data_mining_presentation/data/index. html, 2007.

[14] L.H. Witten and E. Frank, Data Mining: Practical Machine Leaming Tools and Techniques, Morgan Kaufmann, 2005.

[15] Network Associates Inc., http://www.nai.com/, 2006.

[16] Nucleus Research report: "Spam: The Silent ROI Killer," July,

http://www.nucleusresearch.com/research/d59.pdf.

2003,

[17] Paris Trudeau, Richard Cullen and Dave Zwieback, "Major Techniques for Classifying Spam", April 2003.
[18] R. E. Schapire, "The strength of weak learnability," Machine Learning, vol. 5, no. 2 pp 197-227, 1990.

[19] R. Jin, Y. Liu, L. Si, J. Carbonell and A. Hauptmann, "A New Boosting Algorithm Using Input-Dependent Regularizer," in Proceedings of Twentieth International Conference on Machine Learning (ICML'03) 2003.

[20] SurfControl Company, http://www.surfcontrol.com/, 2006. [21] Symantec Corporation, http://www.symantec.com/,

[22] Trend Micro Inc., http://www.trendmicro.com/, 2006.

[23] Tumbleweed Communications Corp http://www.tumbleweed.com/, 2006.

[24] Y. Freund and R. E, Schapire, "A decision theoretic generalization of online learning and an application to boosting", Unpublished manuscript available electronically (by email request). An extended abstract appeared in Computational Learning Theory: Second European Conference, EuroCOLT '95, pp 23-37, Springer-Verlag, 1995.

[25] Y. Freund, "Boosting a weak learning algorithm by majority," Information and Computation, vol.121 no. 2 pp 256-285, 1995.

[26] Y. Freund and R.E. Schapire, "Experiments with a new boosting algorithm," In the Proceeding of International Conference on Machine Learning, pp 148-156, 1996.

[27] Zix Corporation, http://www.zixcorp.com, 2006. 